\title{
Corona Pandemic and herd immunity
}

\section{Assist. Lec. Afkar Fadhil Kareem}

\section{Corona}

In light of the outbreak of the epidemic crisis, as people died, the number of infected people around the world exceeded 9 million, and 469,399 deaths in more than 190 countries.

Because the vaccine has not yet been discovered and there is no treatment for this dangerous pandemic virus, some countries have resorted to apply the herd immunity theory.

is a form of protection is not direct from an infectious disease, and occurs when you acquire a large proportion of society 's immunity to certain infections, either because of injury previously or by vaccination, which provides protection for individuals who have no immunity to the disease ${ }^{[2]}{ }^{[1]}$ If a large proportion of the population possesses immunity to a specific disease, it helps these people not to transmit the disease, and consequently the chains of infection may stop, which leads to stopping or slowing the spread of the disease. ${ }^{[3]}$ The higher the proportion of individuals who have immunity in society, the less likely it is that individuals who do not have immunity will mix with people who transmit the disease, which helps to protect them from infection. ${ }^{[1]}$

Many public health officials and pediatricians cite the so-called "herd" or community immunity in justifying vaccination. The explanation for "herd" immunity is that if a certain percentage of the population is vaccinated, the herd thus protects those who have not received the vaccine.

The theory of "herd immunity" states that a population possess natural immunity for life. There are two reasons why this view is false, that the theory has never been proven true.

This hypothesis was introduced by Hedrich in the 1930s. The theory states that if $68 \%$ of the population is infected with measles (and thus possess lifelong measles immunity)

Vaccines do not confer lifelong immunity and the population that received the vaccines represents a specific population, such as children

For example, we were told that if $97 \%$ of children were vaccinated against measles, the remaining $3 \%$ would be under the protection of the "herd".

In fact, children do not live in private societies, but rather within the adult community. These adults have not received, for many years, decades, or even ever, any of the pneumococcal virus vaccines, tuberculosis, chickenpox, measles, mumps, rubella, diphtheria, tetanus, whooping cough, polio, haemophilus meningitis, and seasonal influenza, H1N1 flu, hepatitis A, B, C, and others.

Automatically, $97 \%$ of vaccine coverage falls to $10-12 \%$, depending on the ratio between adult to infant numbers, so herd theory is nothing more than a myth.

According to a team of researchers at Princeton University in Washington, the herd immunity theory can be successful in societies with a high number of young adults, who are often infected with Covid 19 virus.

In order to reach any level of herd immunity, the proportion of people exposed to the virus must be more than $70 \%$.

Sweden was in a state of global deviation by choosing not to close the country, so the number of deaths due to the virus increased compared to neighboring countries.

It has recorded nearly 3,500 deaths associated with the Coronavirus, and this has raised questions about the number of people who may need death in order to develop the herd 
immunity and a group of doctors advanced at the end of April to condemn Sweden's approach and its officials

The World Health Organization also described herd immunity as a brutal calculation

Where some media indicated that finding an effective vaccine against Covid 19 will take several months, which means that herd immunity is the only alternative approach that allows the economy to grow again and allows countries to reopen the ports in the near future.

\section{References :}

1. The Arabization Coordination Office of the Arab Organization for Education, Culture and Science ( 1441 AH - 2020 CE ). Glossary of Covid Terms-19 (English-French-Arabic) (PDF) (1st edition). Rabat - Morocco (published 6 May 2020 AD ). Page 28. Archived from the original (PDF) on May 22, 2020 CE . It was accessed on May 22, 2020 AD .

2. A "Translation (Herd immunity) in the standardized medical dictionary" . Lebanon Library Publishers . Archived from the original on March 26, 2020 . Accessed on 26 March 2020 .

3. "Translation (Herd immunity) in dictionary". Dictionary site . Archived from the original on March 27, 2020 . Accessed on 26 March 2020 